\title{
A systematic review and meta-analysis of sarcopenia as a prognostic factor in gynecological malignancy
}

Emma R Allanson ${ }^{a}$, Yang Peng ${ }^{b}$, Angela Choi ${ }^{c}$, Sandi Hayes ${ }^{d}$, Monika Janda ${ }^{e}$, Andreas Obermair ${ }^{\mathrm{a}, \mathrm{c},{ }^{*}}$

a Queensland Centre for Gynaecological Cancer, Royal Brisbane and Women's Hospital, Brisbane, Australia

${ }^{b}$ Centre for Clinical Research, The University of Queensland, Brisbane, Australia

c Faculty of Medicine, The University of Queensland, Brisbane, Australia

${ }^{d}$ Menzies Health Institute Queensland, Griffith University, Brisbane, Australia

e Centre of Health Services Research, Faculty of Medicine, The University of Queensland, Brisbane, Australia

*Corresponding author at: Queensland Centre for Gynaecological Cancer,

Centre for Clinical Research, The University of Queensland, Herston QLD 4029, Australia. E-mail address: ao@surgicalperformance.com 


\section{ABSTRACT}

Introduction

Sarcopenia is a condition described as the progressive, generalized loss of muscle mass and strength. While sarcopenia has been linked with poorer outcomes following a variety of malignancies, its relationship with all gynecological cancer clinical outcomes has to date, not been evaluated. This review interrogates the concept of sarcopenia as a prognostic tool for oncological outcomes and adverse effects of treatments in all primary gynecological malignancies.

\section{Methods}

This systematic review and meta-analysis was performed in accordance with the Preferred Reporting Items for Systematic reviews and Meta-Analysis (PRISMA) guidelines, searching PubMed, Embase, and CINAHL without date or language restriction for studies reporting on sarcopenia and gynecological malignancies. Random effects meta-analysis models were used to determine the effects of sarcopenia on progression-free survival, overall survival and treatment-related adverse events.

Results

Data were analysed from 13 studies, including 2446 patients (range 60-323), including those with ovarian cancer $(n=1531)$, endometrial cancer $(n=562)$ or cervical cancer $(n=541)$. Sarcopenia was associated with lower progression free survival (HR: $1.69,95 \% \mathrm{Cl} 1.03$ to 2.76), OS (HR: 1.33 (95\% Cl 1.08 to 1.64) and no increase in adverse events (HR: $1.28,95 \% \mathrm{Cl}$ 0.69 to 2.40 ). The risk of bias of the studies was mostly rated unclear, and Begg's and Egger's test revealed a potential publication bias for progression free survival and OS, although the hazard ratios remained significant when adjusting for it.

Discussion 
Sarcopenia is associated with worse progression free survival and overall survival in gynecological oncology malignancies. Further research is warranted to validate those findings in larger and prospective samples, using standardized methodology and to examine if an intervention could reverse its effect in gynecologic oncology trials.

\section{Precis}

Sarcopenia is increasingly recognised as a pathology that contributes to oncological outcomes. We present a meta-analysis of sarcopenia in gynecological malignancies, showing worse progression free survival and overall survival. Future prospective trials are needed.

\section{Highlights}

1. The overall hazard of disease progression was increased by $69 \%$ for those with sarcopenia.

2. Sarcopenia potentially contributes to worse overall survival, with a pooled hazard ratio of 1.33

3. Sarcopenia should be a factor considered in future prospective trials 


\section{Introduction}

Sarcopenia is a condition or syndrome described as the progressive and generalized loss of muscle mass and strength (1). The prevalence of sarcopenia increases with age, with up to $53 \%$ of individuals in their eighth decade considered sarcopenic and more than 40 million people affected worldwide (1). Sarcopenia can also be acquired as a consequence of several pathological processes, including malignancy (2). The diagnosis of sarcopenia is commonly based on assessment of low muscle mass, strength, and performance (3) or on quantifiable objective measures using various imaging modalities, including computed tomography (CT), magnetic resonance imaging (MRI) and dual energy x-ray absorptiometry (4). Measurement of the third lumbar vertebral area of skeletal muscle on CT imaging is the most frequently used test and has been shown to correlate well with whole body muscle mass (5). Sarcopenia is not reflective of an individual's nutritional status and as such, while it may be more readily diagnosed in normal or underweight individuals (5), body mass index generally correlates poorly with the diagnosis (6) and so sarcopenia can also occur concurrently with obesity, which may be associated with worse outcomes in the context of cancer treatments (7).

Sarcopenia has been shown to independently impact the course of several diseases, including gastrointestinal, renal, and lung malignancies (4). The relationship between sarcopenia and disease outcomes in oncological populations reflects an emerging area of research, and assessment of the impact of sarcopenia on morbidity and mortality in gynecological oncology is therefore a growing area of study (8). Traditionally, gynecologic oncologists have placed great emphasis on the detection and management of cachexia (weight loss), as its presence is associated with adverse malignancy-associated outcomes 
(9). It is clear however that body mass index is not correlated with body composition and knowledge of muscle mass, or lack thereof, is an increasingly important consideration in the treatment of malignancy (10). This may be of particular relevance in gynecological oncology given the strong association between obesity and risk of endometrial and ovarian malignancies (11), where there will be a need for improved understanding of the less clinically apparent, sarcopenia in overweight and obese patients. Patient factors which allow clinicians to refine their treatment and prognostication are increasingly important in an era of personalized medicine. Sarcopenia may provide clinical information that allow treatment interventions not as readily apparent if one relies on more traditional assessments of nutrition (e.g. body mass index)(12), and further research is necessary to assess whether this process is of oncological benefit.

Thus this meta-analysis and systematic review of the literature aims to interrogate the concept of sarcopenia as a prognostic tool for oncological outcomes and for its association with treatment-related complications in the context of gynecological cancer.

\section{Methods}

This systematic review and meta-analysis was performed in accordance with the Preferred Reporting Items for Systematic reviews and Meta-Analysis guidelines. We searched PubMed, Embase, and CINAHL to identify all potentially relevant articles with no date limitation, without language restriction. Search strategies were customized to the subject heading and search structure of individual databases. Reference lists from review articles were also searched. The full search strategy can be found in Appendix 1. The search was 
completed on the $25^{\text {th }}$ of June, 2019. This study was registered with PROSPERO, registration number CRD42019139488.

\section{Inclusion and exclusion criteria}

All identified studies were assessed. Any study reporting on sarcopenia and outcomes in gynecologic cancer was considered, with participants in studies defined as women with uterine/endometrial, ovarian, cervical, vulvar, or vaginal cancer. Sarcopenia was defined using an accepted objective measure as determined by the study authors (see results). Cohort studies (prospective and retrospective) and randomized controlled trials were considered for inclusion. Conference abstracts, case reports and management recommendations or pharmaceutical treatment studies were excluded. Studies were excluded if they didn't report on the pre-determined outcome measures of interest and / or sarcopenia was not defined.

\section{Outcome measures}

Pre-determined outcomes of interest were progression-free survival, as defined as time from enrollment in the study until recurrence of disease; overall survival as defined as time from enrollment in the study until death from any cause; and adverse events associated with treatment, which were defined by the study authors and included intra-operative and post-operative complications, as well as complications of chemotherapy (Appendix 2). We also targeted tolerability of treatment as an outcome measure, however no study included in the final meta-analysis reported on this.

\section{Selection of studies}


Two authors (AC and YP) independently reviewed abstracts to identify all studies that potentially met the inclusion criteria and should be retrieved. The same two authors independently assessed each full text article to determine whether it met all of the selection criteria. Any disagreement and uncertainties were resolved by discussion, with involvement of a third author (EA).

\section{Assessment of methodological and reporting quality}

Methodological quality was assessed using the Cochrane Risk of Bias tool for randomized trials. For non-randomised studies, the Newcastle-Ottawa Scale was adapted and used for the cohort studies as it included three additional elements (all outcome measures reported, methods of assessment for outcome provided, authors discuss potential sources of bias) from the Strengthening the Reporting of Observational Studies in Epidemiology quality assessment tool when assessing cohort studies.

\section{Statistical analysis}

Study results were summarised in descriptive form, including number of patients involved, study design, year of study and main outcome measures. We conducted a meta-analysis of the combined data across all studies, regardless of cancer type first. Given the heterogeneity of the included studies, pooled hazard ratios were estimated using randomeffects. To determine the effects of sarcopenia on progression free survival, overall survival, and adverse events we calculated the pooled hazard ratios (HR) and their corresponding 95\% confidence intervals ( $\mathrm{Cl})$. A two-tailed $P$ value $<0.05$ was considered statistically significant. Sarcopenia was defined according to the authors of individual studies. Given the range of accepted diagnostic modalities it was pragmatic to analyse studies together and 
accept the limitations of this. We used Begg's and Egger's tests to evaluate the publication bias, with a two-tailed $P<0.1$ indicating significant publication bias. All analyses were conducted using Stata software, version 15.1 (Stata Corp, College Station, TX, USA).

\section{Results}

Fig. 1 shows the Preferred Reporting Items for Systematic reviews and Meta-Analysis flow chart for the selection of studies. The search strategy returned 342 results after duplicates were removed. Of these, after exclusions were applied, 51 full texts were reviewed (14.9\%), with 16 studies meeting the inclusion criteria.

The characteristics of the 16 included studies, of which all were retrospective cohort studies, are shown in Table 1. Nine studies (56.25\%) reported on sarcopenia in ovarian cancer, $3(18.75 \%)$ on endometrial cancer, 1 (6.35\%) on both endometrial and ovarian cancer (with data combined), and 3 (18.75\%) on cervical cancer. Sample sizes ranged from 60 to 323 patients. Three studies(13-15) had crossover periods and potential crossover patients with other included papers and so were excluded from any further analysis. 2466 patients from 13 studies were included in the final meta-analysis. All included studies were published from 2015 onwards. Sarcopenia was defined in a variety of ways including muscle attenuation (16), skeletal muscle index $(9,14,17-25)$, muscle mass measurement (26), skeletal muscle loss during treatment $(13,15)$, and the psoas index $(27)$, all of which were defined in the included papers. Ten (77\%) studies reported on overall survival, 4 (31\%) reported on progression free survival, and $5(38 \%)$ studies reported on adverse events (Appendix 2). 
Progression free survival

2 Overall, 4 studies ( 2 ovarian and 1 each for endometrial and cervical cancer) contributed

3 data towards analysis exploring the relationship between sarcopenia and disease

4 progression. The progression free survival by cancer type and overall is shown in Fig. 2.

5 Findings indicate that overall the hazard of disease progression was increased by $69 \%$ for

6 those with sarcopenia (HR: $1.69,95 \% \mathrm{Cl} 1.03$ to $2.76, I^{2} 54.6 \%, P=0.085$; low to moderate

7 heterogeneity in the included studies). The relationship between progression free survival

8 and sarcopenia is inverse, which means that the presence of sarcopenia has a negative

9 impact on progression free survival.

\section{Overall survival}

12 Ten studies reported on the relationship between sarcopenia and overall survival. The

13 overall pooled hazard ratio was $1.33\left(95 \% \mathrm{Cl} 1.08\right.$ to $\left.1.64, I^{2} 40.8 \%, \mathrm{P}=0.086\right)$ with low to

14 moderate heterogeneity in the included studies. Subgroup analyses showed the magnitude

15 of effect is accentuated for women with endometrial cancer (Fig. 3). The relationship

16 between overall survival and sarcopenia is inverse, with the presence of sarcopenia

17 associated with a negative impact on overall survival.

Adverse events

Data from 5 studies (with high heterogeneity) suggest no significant increase in adverse events in those with sarcopenia (HR: $1.28,95 \% \mathrm{Cl} 0.69$ to $2.40, \mathrm{I}^{2}=69.4 \%, P=0.011$ ) (Fig. 4). 
24 Supplemental Fig. 1 summarises the assessment of methodological quality of the included 25 studies. The majority of studies had an unclear risk of bias in seven of eight domains. Six of 2613 studies (46\%) had a high risk of bias in the domain of representativeness of the exposed 27 cohort. Six studies were also at low risk of bias in the domain of comparability of the 28 cohorts.

Publication bias

Publication bias was assessed using both Begg's and Egger's tests. For OVERALL SURVIVAL potential publication bias was identified (Begg's test $p$-value $=0.049$, Egger's test $p$-value $=$ 0.014), however the HR remained significant after applying the trim and fill method $(H R=$ $1.21,95 \% \mathrm{Cl} 1.03-1.42)$. This was similar in progression free survival (Begg's test $\mathrm{p}$-value $=$ 0.089 , Egger's test $p$-value $=0.063)$, with a HR after applying the trim and fill method of 1.54 (95\% $\mathrm{Cl} 1.07-2.18)$. There was no apparent publication bias for the outcome of adverse events (Begg's test $p$-value $=0.324$, Egger's test $p$-value $=0.115, H R=1.30,95 \% \mathrm{Cl} 0.72-2.37$ )

\section{Discussion}

This systematic review and meta-analysis explored the relationship between sarcopenia and gynecologic oncology outcomes. We found evidence from 13 studies that sarcopenia was significantly associated with impaired overall survival and progression free survival with a trend towards an increased risk of adverse events. The impact of sarcopenia on overall survival was pronounced for patients treated for endometrial cancer. Sarcopenia was also associated with a trend towards higher adverse events risk related to cancer treatment

46 (variably defined), although these results should be interpreted with caution as only few 
47 studies reported on this outcome, and there was marked heterogeneity of the included studies as indicated by the high I-index (69.4\%).

Prior to this review, the relevant body of evidence for gynecologic malignancies consisted of only one meta-analysis, including data from 8 studies, suggesting a significant association of low skeletal muscle index with overall survival in ovarian cancer (HR 1.11). However, the authors concluded that the quality of the evidence was low in all contributing publications

54 (28). In our meta-analyses, there is a trend towards worse overall survival in sarcopenia in ovarian cancer (6 studies) and a significant association between sarcopenia and overall survival endometrial cancer ( 2 publications). Further, although lacking statistical significance, findings relevant to women with cervical cancer were in the same direction as those for women with endometrial and ovarian cancer. The magnitude of effect reported from this review was consistent with those from a variety of other solid malignancies, including gastric (29), renal and colorectal malignancies (30).

In our analysis of progression free survival, there were small numbers of included studies but overall a significant association of sarcopenia with progression free survival. In other oncological studies, there are conflicting results with regards to the impact of sarcopenia on this outcome (30). Given the small number of studies, the role of sarcopenia in specific gynecological malignancies is difficult to interrogate. It is however apparent that sarcopenia has the potential to be of significance in the progression of both ovarian and endometrial malignancies and it will be important to explore the mechanisms driving this effect in future prospective studies. 
71 Although less well studied, in a variety of solid malignancies such as gastric and

72 hepatopancreatobiliary tumors, sarcopenia has been associated with increased treatment-

73 related complications $(31,32)$. We did not find a significant relationship between sarcopenia

74 and adverse events in this analysis, although included studies and numbers of patients were

75 limited.

76

77 Of particular note are the findings of this meta-analysis pertaining to women with endometrial cancer. Specifically, compared with the overall HR across all gynaecological malignancies, HR relating to subgroup analysis for overall survival following endometrial cancer was the most pronounced. Over $60 \%$ of women diagnosed with endometrial cancer are obese (33), and the diagnosis of sarcopenia in obese patients may be challenging.

82 Assessment of sarcopenia prior to treatment could assist with identification of patients at high risk of poor outcomes. Those patients could be targeted for future intervention trials.

This systematic review and meta-analysis had broad inclusion criteria and followed rigorous quality protocols. All papers included were published from 2015 onwards, highlighting these findings as likely reflective of current patients and practice. Nonetheless, key limitations of this review include the heterogeneous methods of sarcopenic assessment and retrospective data collection of included studies, with the latter limitation likely contributing to unclear risk of bias and the publication bias identified. The accepted definitions and specific measurement of sarcopenia varied widely between studies, which limits the interpretation

92 of the analysis and the capacity to rigorously compare included studies. We were unable to 93 interrogate the impact of sarcopenia diagnosed at different time points in the course of 94 cancer treatment, and are aware that this may impact the magnitude of the effect of 
sarcopenia on patient outcomes(34). A standardized approach to the diagnosis will be

necessary if we are to consider further the role it may play in trials and assessment of

oncological outcomes. Furthermore, while sarcopenia may be acquired as a consequence of cancer treatment, for example as a side-effect of chemotherapy(35), it is also clear that it may associated with age, previous comorbidities and declines in physical activity(1).

Retrospective studies, such as those included in this meta-analysis, lack the methodological robustness to provide sufficient detail regarding the direction of the relationship being assessed.

There is also clear scope for rigorous and comprehensive assessment the potential relationship between adverse events and sarcopenia in gynecological malignancies. It was beyond the scope of this review to evaluate the timing of sarcopenia development and its

107 impact on survival outcomes and adverse events; this represents a clear area in need of

108 future research with high translational potential. The lack of prospective studies in this field remains a significant limitation in being able to comprehensively consider the role of

110 sarcopenia in clinical gynecological oncology, particularly with respect to both its capacity to

111 be modified as well as its potential to guide prognosis at various stages of an oncological

112 diagnosis. Therefore, future prospective research with a uniform definition of sarcopenia 113 and with consideration to the relationship between sarcopenia and other prognostic factors

114 in malignancy is required to obtain high-level evidence on the impact of sarcopenia on 115 treatment-related complications and survival outcomes.

117 In conclusion, sarcopenia is associated with worse oncological outcomes in gynecological 118 malignancies in this systematic review of retrospective cohort studies. Given that sarcopenia 
119 is a potentially modifiable risk factor, prospective research using predefined criteria is

120 warranted to explore the potential of interventions to reverse it.

121

122

\section{Conflict of interest}

124 The authors declare no conflict of interest.

\section{Acknowledgements}

126 We would like to acknowledge Mr. Lars Eriksson, the liaison librarian for the Faculty of

127 Medicine at the University of Queensland for assistance with development of the search

128 strategy.

129 Funding

$130 \quad$ No funding source to declare.

\section{Author contributions}

132 Study design: Emma R Allanson, Andreas Obermair.

133 Data acquisition: Emma R Allanson, Yang Peng, Angela Choi.

134 Quality assessment: Emma R Allanson, Yang Peng, Angela Choi.

135 Data analysis and interpretation: Yang Peng, Sandi Hayes, Monika Janda.

136 Manuscript draft: Emma R Allanson.

137 Manuscript review: Emma R Allanson, Yang Peng, Angela Choi, Sandi Hayes, Monika

138 Janda, Andreas Obermair.

139 All authors approved the final version of the manuscript.

140

141 
143 1. Santilli V, Bernetti A, Mangone M, Paoloni M. Clinical definition of sarcopenia. Clinical cases in mineral and bone metabolism. 2014;11(3):177.

145 2. Bauer J, Morley JE, Schols AM, Ferrucci L, Cruz - Jentoft AJ, Dent E, et al. Sarcopenia: A Time for Action. An SCWD Position Paper. Journal of cachexia, sarcopenia and muscle. 2019.

148 3. Cruz-Jentoft AJ, Baeyens JP, Bauer JM, Boirie Y, Cederholm T, Landi F, et al.

149 Sarcopenia: European consensus on definition and diagnosisReport of the European Working Group on Sarcopenia in Older PeopleA. J. Cruz-Gentoft et al. Age Ageing. 2010;39(4):412-23.

4. Marty E, Liu Y, Samuel A, Or O, Lane J. A review of sarcopenia: Enhancing awareness of an increasingly prevalent disease. Bone. 2017;105:276-86.

154 5. Cruz-Jentoft AJ, Bahat G, Bauer J, Boirie Y, Bruyère 0 , Cederholm $T$, et al. Sarcopenia: revised European consensus on definition and diagnosis. Age Ageing. 2018;48(1):16-31. 6. Baracos VE, Arribas L. Sarcopenic obesity: hidden muscle wasting and its impact for survival and complications of cancer therapy. Ann Oncol. 2018;29(suppl_2):ii1-ii9. 7. Hilmi M, Jouinot A, Burns R, Pigneur F, Mounier R, Gondin J, et al. Body composition and sarcopenia: The next-generation of personalized oncology and pharmacology? Pharmacol Ther. 2019;196:135-59.

162

163

164

165 8. Heard RSM, Ramsay G, Hildebrand DR. Sarcopaenia in surgical populations: A review. Surgeon. 2017;15(6):366-71.

9. Bronger H, Hederich P, Hapfelmeier A, Metz S, Noel PB, Kiechle M, et al. Sarcopenia in Advanced Serous Ovarian Cancer. Int J Gynecol Cancer. 2017;27(2):22332.

167 10. Caan BJ, Cespedes Feliciano EM, Kroenke CH. The Importance of Body

168 Composition in Explaining the Overweight Paradox in Cancer-Counterpoint. Cancer Res. 169 2018;78(8):1906-12.

170 11. Kyrgiou M, Kalliala I, Markozannes G, Gunter MJ, Paraskevaidis E, Gabra H, et al. 171 Adiposity and cancer at major anatomical sites: umbrella review of the literature. BMJ. $172 \quad$ 2017;356:j477.

173 12. Prado CM, Purcell SA, Laviano A. Nutrition interventions to treat low muscle 174 mass in cancer. J Cachexia Sarcopenia Muscle. 2020;11(2):366-80.

175 13. Kiyotoki T, Nakamura K, Haraga J, Omichi C, Ida N, Saijo M, et al. Sarcopenia Is an 176 Important Prognostic Factor in Patients With Cervical Cancer Undergoing Concurrent 177 Chemoradiotherapy. Int J Gynecol Cancer. 2018;28(1):168-75.

178 14. Rodrigues CS, Chaves GV. Skeletal Muscle Quality Beyond Average Muscle 179 Attenuation: A Proposal of Skeletal Muscle Phenotypes to Predict Short-Term Survival 180 in Patients With Endometrial Cancer. J Natl Compr Canc Netw. 2018;16(2):153-60.

181 15. Rutten IJG, Ubachs J, Kruitwagen R, Beets-Tan RGH, Olde Damink SWM, Van Gorp 182 T. Psoas muscle area is not representative of total skeletal muscle area in the 183 assessment of sarcopenia in ovarian cancer. J Cachexia Sarcopenia Muscle. 184 2017;8(4):630-8.

185 16. Ataseven B, Luengo TG, du Bois A, Waltering KU, Traut A, Heitz F, et al. Skeletal 186 Muscle Attenuation (Sarcopenia) Predicts Reduced Overall Survival in Patients with 187 Advanced Epithelial Ovarian Cancer Undergoing Primary Debulking Surgery. Ann Surg 188 Oncol. 2018;25(11):3372-9. 

Depletion and Markers for Cancer Cachexia Are Strong Prognostic Factors in Epithelial Ovarian Cancer. PLoS One. 2015;10(10):e0140403.

192 18. Conrad LB, Awdeh H, Acosta-Torres S, Conrad SA, Bailey AA, Miller DS, et al. Preoperative core muscle index in combination with hypoalbuminemia is associated with poor prognosis in advanced ovarian cancer. J Surg Oncol. 2018;117(5):1020-8.

195 19. Silva de Paula N, de Aguiar Bruno K, Azevedo Aredes M, Villaca Chaves G. Sarcopenia and Skeletal Muscle Quality as Predictors of Postoperative Complication and Early Mortality in Gynecologic Cancer. Int J Gynecol Cancer. 2018;28(2):412-20. 20. de Paula NS, Rodrigues CS, Chaves GV. Comparison of the prognostic value of different skeletal muscle radiodensity parameters in endometrial cancer. Eur J Clin Nutr. 2019;73(4):524-30.

21. Kumar A, Moynagh MR, Multinu F, Cliby WA, McGree ME, Weaver AL, et al. Muscle composition measured by CT scan is a measurable predictor of overall survival in advanced ovarian cancer. Gynecol Oncol. 2016;142(2):311-6.

22. Lee J, Chang CL, Lin JB, Wu MH, Sun FJ, Jan YT, et al. Skeletal Muscle Loss Is an Imaging Biomarker of Outcome after Definitive Chemoradiotherapy for Locally Advanced Cervical Cancer. Clin Cancer Res. 2018;24(20):5028-36.

23. Matsuoka H, Nakamura K, Matsubara Y, Ida N, Nishida T, Ogawa C, et al. Sarcopenia Is Not a Prognostic Factor of Outcome in Patients With Cervical Cancer Undergoing Concurrent Chemoradiotherapy or Radiotherapy. Anticancer Res. 2019;39(2):933-9.

24. Rutten IJ, Ubachs J, Kruitwagen RF, van Dijk DP, Beets-Tan RG, Massuger LF, et al. The influence of sarcopenia on survival and surgical complications in ovarian cancer patients undergoing primary debulking surgery. Eur J Surg Oncol. 2017;43(4):717-24. 25. Rutten IJ, van Dijk DP, Kruitwagen RF, Beets-Tan RG, Olde Damink SW, van Gorp T. Loss of skeletal muscle during neoadjuvant chemotherapy is related to decreased survival in ovarian cancer patients. J Cachexia Sarcopenia Muscle. 2016;7(4):458-66. 26. Kuroki LM, Mangano M, Allsworth JE, Menias CO, Massad LS, Powell MA, et al. Pre-operative assessment of muscle mass to predict surgical complications and prognosis in patients with endometrial cancer. Ann Surg Oncol. 2015;22(3):972-9. 27. Yoshikawa T, Takano M, Miyamoto M, Yajima I, Shimizu Y, Aizawa Y, et al. Psoas muscle volume as a predictor of peripheral neurotoxicity induced by primary chemotherapy in ovarian cancers. Cancer Chemother Pharmacol. 2017;80(3):555-61.

223 28. Ubachs J, Ziemons J, Minis - Rutten IJ, Kruitwagen RF, Kleijnen J, Lambrechts S, et

224 al. Sarcopenia and ovarian cancer survival: a systematic review and meta - analysis. Journal of cachexia, sarcopenia and muscle. 2019. 29. Sierzega M, Chrzan R, Wiktorowicz M, Kolodziejczyk P, Richter P. Prognostic and predictive implications of sarcopenia in Western patients undergoing gastric resections for carcinoma of the stomach. J Surg Oncol. 2019;120(3):473-82.

30. Shachar SS, Williams GR, Muss HB, Nishijima TF. Prognostic value of sarcopenia in adults with solid tumours: A meta-analysis and systematic review. Eur J Cancer. 2016;57:58-67.

31. Hua H, Xu X, Tang Y, Ren Z, Xu Q, Chen L. Effect of sarcopenia on clinical outcomes following digestive carcinoma surgery: a meta-analysis. Support Care Cancer. 2019;27(7):2385-94. 
235 32. Cao Q, Xiong Y, Zhong Z, Ye Q. Computed Tomography-Assessed Sarcopenia 236 Indexes Predict Major Complications following Surgery for Hepatopancreatobiliary 237 Malignancy: A Meta-Analysis. Ann Nutr Metab. 2019;74(1):24-34.

238 33. Janda M, Gebski V, Davies LC, Forder P, Brand A, Hogg R, et al. Effect of Total 239 Laparoscopic Hysterectomy vs Total Abdominal Hysterectomy on Disease-Free Survival 240 Among Women With Stage I Endometrial Cancer: A Randomized Clinical Trial. JAMA. 241 2017;317(12):1224-33.

242 34. Le-Rademacher JG, Storrick EM, Jatoi A. Remarks on the design and analyses of 243 longitudinal studies for cancer patients with anorexia and weight loss. J Cachexia 244 Sarcopenia Muscle. 2019;10(6):1175-82.

245 35. Davis MP, Panikkar R. Sarcopenia associated with chemotherapy and targeted 246 agents for cancer therapy. Ann Palliat Med. 2019;8(1):86-101.

247 36. de Paula NS, de Aguiar Bruno K, Aredes MA, Chaves GV. Sarcopenia and skeletal 248 muscle quality as predictors of postoperative complication and early mortality in 249 gynecologic cancer. International Journal of Gynecologic Cancer. 2018;28(2):412-20. 
251 Table 1. General characteristics of included studies, all of which were retrospective cohort studies.

\begin{tabular}{|c|c|c|c|c|c|c|c|c|c|}
\hline First author & $\begin{array}{c}\text { Publication } \\
\text { year }\end{array}$ & Country & $\begin{array}{c}\text { Study period } \\
\text { (year) }\end{array}$ & Cancer type & Sample size & $\begin{array}{c}\text { Median follow-up } \\
\text { (months) }\end{array}$ & Age (years) & Sarcopenia criteria & Outcome \\
\hline Ataseven B (16) & 2018 & Germany & 2011-2016 & Ovarian & 323 & 40 & $\begin{array}{l}\text { Median 60, } \\
\text { range 21-89 }\end{array}$ & $\begin{array}{c}\text { Muscle attenuation } \\
<32 \mathrm{HU}\end{array}$ & Overall survival \\
\hline Aust S (17) & 2015 & Austria & 2004-2012 & Ovarian & 140 & 56 & Mean 60 & $\begin{array}{c}\text { Skeletal muscle } \\
\text { index }<41 \mathrm{~cm} 2 / \mathrm{m} 2\end{array}$ & $\begin{array}{c}\text { Progression free } \\
\text { survival; Overall } \\
\text { survival }\end{array}$ \\
\hline Bronger H (9) & 2017 & Germany & $2003-2013$ & Ovarian & 105 & 27 & $\begin{array}{l}\text { Median } 65, \\
\text { range } 33-85\end{array}$ & $\begin{array}{c}\text { Skeletal muscle } \\
\text { index } \leq 38.5 \\
\mathrm{~cm} 2 / \mathrm{m} 2\end{array}$ & $\begin{array}{c}\text { Progression free } \\
\text { survival; Overall } \\
\text { survival }\end{array}$ \\
\hline Conrad LB (18) & 2017 & US & $2007-2015$ & Ovarian & 102 & 26 & Mean 55 & $\begin{array}{c}\text { Skeletal muscle } \\
\text { index }<38.5 \\
\mathrm{~cm} 2 / \mathrm{m} 2\end{array}$ & Adverse events \\
\hline de Paula NS (36) & 2018 & Brazil & $2008-2015$ & $\begin{array}{c}\text { Endometrial } \\
\text { Ovarian }\end{array}$ & 250 & 1 & Not available & $\begin{array}{c}\text { Skeletal muscle } \\
\text { index }<38.9\end{array}$ & Adverse events \\
\hline de Paula NS (20) & 2019 & Brazil & $2008-2015$ & Endometrial & 232 & 12 & Median 64.3 & $\begin{array}{c}\text { Skeletal muscle } \\
\text { index }<38.9 \\
\mathrm{~cm} 2 / \mathrm{m} 2\end{array}$ & Overall survival \\
\hline Kiyotoki T (13) & 2018 & Japan & 2004-2014 & Cervical & 60 & 33.5 & $\begin{array}{c}\text { Median 56.1, } \\
\text { range 25-74 }\end{array}$ & $\begin{array}{c}\text { Skeletal muscle } \geq \\
15 \% \text { loss during } \\
\text { treatment }\end{array}$ & $\begin{array}{c}\text { Progression free } \\
\text { survival; Overall } \\
\text { survival }\end{array}$ \\
\hline Kumar A (21) & 2016 & US & $2006-2012$ & Ovarian & 296 & 33.2 & Mean 64.6 & $\begin{array}{c}\text { Skeletal muscle } \\
\text { index }<39 \mathrm{~cm} 2 / \mathrm{m} 2\end{array}$ & Overall survival \\
\hline Kuroki LM (26) & 2015 & US & $2005-2009$ & Endometrial & 122 & 32.8 & Mean 65.9 & $\begin{array}{c}\text { Muscle mass }<4.33 \\
\mathrm{~cm} 2\end{array}$ & $\begin{array}{c}\text { Progression free } \\
\text { survival; Overall } \\
\text { survival; } \\
\text { Adverse events }\end{array}$ \\
\hline
\end{tabular}




\begin{tabular}{|c|c|c|c|c|c|c|c|c|c|}
\hline Lee J (22) & 2018 & Taiwan & $2004-2015$ & Cervical & 245 & 62.7 & Mean 63 & $\begin{array}{c}\text { Skeletal muscle } \\
\text { index }<41 \mathrm{~cm} 2 / \mathrm{m} 2\end{array}$ & Overall survival \\
\hline $\begin{array}{l}\text { Matsuoka H } \\
\text { (23) }\end{array}$ & 2019 & Japan & $2004-2018$ & Cervical & 236 & 34.5 & $\begin{array}{l}\text { Median } 61, \\
\text { range } 25-88\end{array}$ & $\begin{array}{c}\text { Skeletal muscle } \\
\text { index }<36.55 \\
\mathrm{~cm} 2 / \mathrm{m} 2\end{array}$ & $\begin{array}{c}\text { Progression free } \\
\text { survival; Overall } \\
\text { survival }\end{array}$ \\
\hline $\begin{array}{l}\text { Rodrigues CS } \\
\text { (14) }\end{array}$ & 2018 & Brazil & $2008-2014$ & Endometrial & 208 & 12 & Mean 64.2 & $\begin{array}{c}\text { Skeletal muscle } \\
\text { index }<42.4 \\
\mathrm{~cm} 2 / \mathrm{m} 2 \\
\text { Average muscle } \\
\text { radiation } \\
\text { attenuation }<30 \mathrm{HU}\end{array}$ & Overall survival \\
\hline Rutten IJG (25) & 2016 & Netherlands & $2000-2014$ & Ovarian & 123 & 23 & $\begin{array}{l}\text { Mean } 66.5, \\
\text { range } 39-86\end{array}$ & $\begin{array}{c}\text { Skeletal muscle } \\
\text { index }<41.5 \\
\mathrm{~cm} 2 / \mathrm{m} 2\end{array}$ & Overall survival \\
\hline Rutten IJG (24) & 2017 & Netherlands & $2000-2015$ & Ovarian & 216 & 57 & $\begin{array}{l}\text { Mean 63.1, } \\
\text { range } 16-85\end{array}$ & $\begin{array}{c}\text { Skeletal muscle } \\
\text { index } \leq 38.73 \\
\mathrm{~cm} 2 / \mathrm{m} 2\end{array}$ & $\begin{array}{l}\text { Overall survival; } \\
\text { Adverse events }\end{array}$ \\
\hline Rutten IJG (15) & 2017 & Netherlands & $2000-2015$ & Ovarian & 150 & 23 & $\begin{array}{l}\text { Median } 67, \\
\text { range } 39-86\end{array}$ & $\begin{array}{l}\text { Skeletal muscle } \\
\text { attenuation loss } \\
\text { during treatment }\end{array}$ & Overall survival \\
\hline $\begin{array}{l}\text { Yoshikawa T } \\
\text { (27) }\end{array}$ & 2017 & Japan & 2010-2015 & Ovarian & 76 & NA & $\begin{array}{l}\text { Median 62, } \\
\text { range } 33-81\end{array}$ & $\begin{array}{c}\text { Psoas index }<58 \\
\mathrm{~mm} 2 / \mathrm{m} 2\end{array}$ & Adverse events \\
\hline
\end{tabular}




\section{Table/Figure legends}

255 Table 1. General characteristics of included studies, all of which were retrospective cohort 256 studies.

257 Fig.1. Preferred Reporting Items for Systematic reviews and Meta-Analysis checklist of 258 included studies.

259 Fig.2. The forest plot for association between sarcopenia and progression-free survival. HR, 260 hazard ratio; $\mathrm{Cl}$, confidence interval.

261 Fig.3. The forest plot for association between sarcopenia and overall survival. HR, hazard 262 ratio; $\mathrm{Cl}$, confidence interval.

263 Fig.4. The forest plot for association between sarcopenia and adverse events. HR, hazard 264 ratio; $\mathrm{Cl}$, confidence interval. 